\title{
A scintillator-based approach to monitor secondary neutron production during proton therapy
}

\author{
S. D. Clarke, ${ }^{\text {a) }}$ E. Pryser, B. M. Wieger, and S. A. Pozzi \\ Department of Nuclear Engineering and Radiological Sciences, University of Michigan, \\ 2355 Bonisteel Boulevard, Ann Arbor, Michigan 48109 \\ R. A. Haelg \\ Center for Proton Therapy, Paul Scherrer Institut, Villigen PSI West 5232, Switzerland \\ V. A. Bashkirov and R. W. Schulte \\ Division of Radiation Research, Department of Basic Sciences, Loma Linda University, 11021 Campus Street, \\ Loma Linda, California 92354
}

(Received 24 February 2016; revised 12 September 2016; accepted for publication

18 September 2016; published 10 October 2016)

Purpose: The primary objective of this work is to measure the secondary neutron field produced
by an uncollimated proton pencil beam impinging on different tissue-equivalent phantom materials
using organic scintillation detectors. Additionally, the Monte Carlo code McNPX-PoliMi was used to
simulate the detector response for comparison to the measured data. Comparison of the measured
and simulated data will validate this approach for monitoring secondary neutron dose during proton
therapy. Methods: Proton beams of $155-$ and $200-\mathrm{MeV}$ were used to irradiate a variety of phantom materials and secondary particles were detected using organic liquid scintillators. These detectors are sensitive to fast neutrons and gamma rays: pulse shape discrimination was used to classify each detected pulse as either a neutron or a gamma ray. The MCNPX-PoliMi code was used to simulate the secondary neutron field produced during proton irradiation of the same tissue-equivalent phantom materials.

Results: An experiment was performed at the Loma Linda University Medical Center proton therapy research beam line and corresponding models were created using the McNPX-PoliMi code. The authors' analysis showed agreement between the simulations and the measurements. The simulated detector response can be used to validate the simulations of neutron and gamma doses on a particular beam line with or without a phantom.

Conclusions: The authors have demonstrated a method of monitoring the neutron component of the secondary radiation field produced by therapeutic protons. The method relies on direct detection of secondary neutrons and gamma rays using organic scintillation detectors. These detectors are sensitive over the full range of biologically relevant neutron energies above $0.5 \mathrm{MeV}$ and allow effective discrimination between neutron and photon dose. Because the detector system is portable, the described system could be used in the future to evaluate secondary neutron and gamma doses on various clinical beam lines for commissioning and prospective data collection in pediatric patients treated with proton therapy. (ㅇ 2016 American Association of Physicists in Medicine. [http://dx.doi.org/10.1118/1.4963813]

Key words: proton therapy; dosimetry; neutron dose; Monte Carlo

\section{INTRODUCTION}

Proton therapy facilities use high-energy protons in place of the more traditional photons or electrons to treat cancer. Protons are recognized for highly conformal dose distributions that improve local tumor control while reducing normal tissue toxicity by limiting unwanted dose. In theory, patients treated with protons should have a drastically lowered risk for radiation-induced secondary malignancies. However, there is concern that high-energy neutrons produced from the inelastic scattering of protons within the treatment head and within the patient may reduce this benefit by depositing unwanted dose outside of the target volume.

Because young patients are more sensitive to radiation, and thus more likely to develop secondary malignancies upon radiation exposure, ${ }^{1}$ proton therapy has been considered superior to photon therapy in the treatment of pediatric patients. The reduced dose to normal tissue offered by protons would, in theory, reduce the number of secondary cancers seen in pediatric patients. However, the presence of the secondary radiation field, mostly due to secondary neutrons and gamma rays, suggests that there may be an additional small risk of secondary cancers that needs to be considered. Additionally, a case study performed by Dorr and colleagues determined that $50 \%$ of second malignancies occurred within a $5 \mathrm{~cm}$ margin surrounding the treatment field, while less than $10 \%$ of secondary malignancies occurred inside of the field. ${ }^{2}$ Hence, it will be critical to evaluate the benefits of proton therapy, specifically for pediatric cases, with carefully designed prospective studies that include evaluation of the 


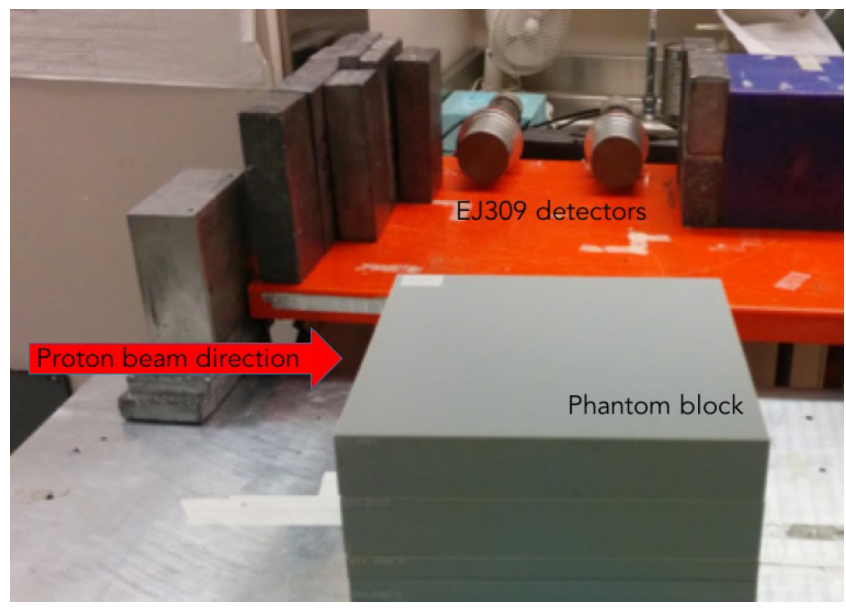

FIG. 1. Photograph of the experimental setup showing the two EJ-309 scintillation detectors on the table with the associated shielding; a phantom block is visible at the bottom of the picture. The proton beam direction is left-to-right.

secondary radiation field. Because of their relatively high biological impact, the neutron component of the field is of particular interest.

Rem-meters, often based on neutron moderation, generally have low sensitivity to neutrons with energies greater than $15 \mathrm{MeV}^{3}$ Additionally, even advanced neutron dosimeters underestimate doses from neutrons less than $2 \mathrm{MeV} .{ }^{4}$ Because the biological effectiveness of neutrons is strongly dependent on their energy, and because the energy spectrum of secondary neutrons extends to the energy of the incident proton beam, rem-meters are inadequate for neutron monitoring purposes in this environment. Accurate neutron monitoring in a proton therapy facility necessitates a detector that can perform active, fast neutron spectroscopy up to energies of $250 \mathrm{MeV}$ and can discriminate between their neutrons and associated gamma rays. Furthermore, the detectors used in this work are sensitive to both neutrons and gamma rays, which can enable monitoring of the dose from the complete secondary radiation field.

From the patient perspective, the neutron dose deposited in normal tissues is of primary importance. This dose is primarily

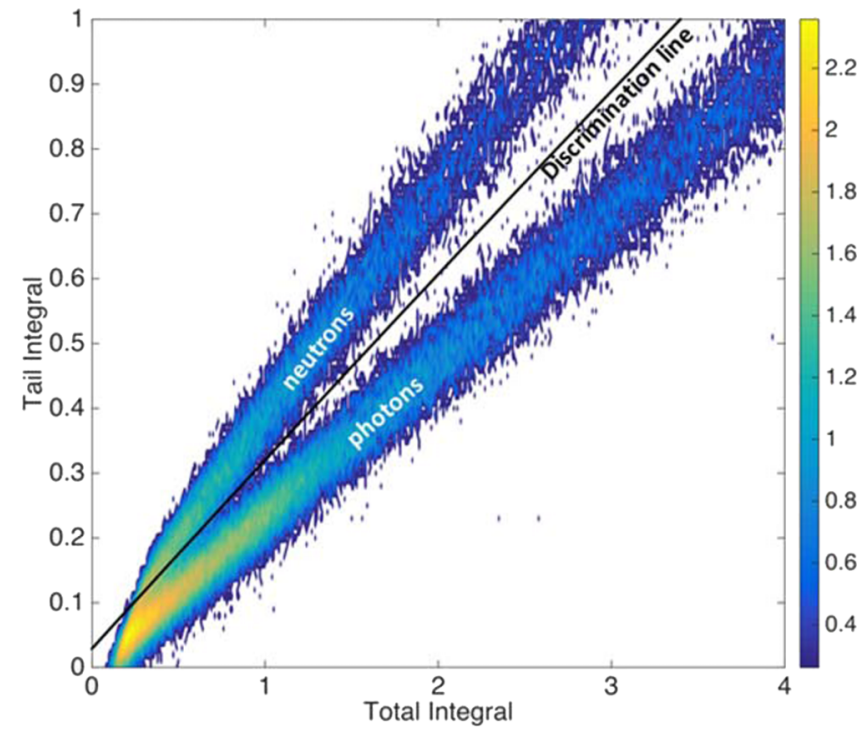

FIG. 2. Pulse shape discrimination results from a soft tissue phantom irradiated with 200-MeV protons; the color bar is logarithmic.

from external neutrons produced in the treatment head as well as internal neutrons produced in the patient. In practice, secondary dose evaluation inside the patient is best done with a Monte Carlo simulation combined with a digital phantom used as a patient surrogate. Modern hybrid phantoms ${ }^{5}$ can be deformed to create an age- and weight-adjusted model of a specific patient that is matched to the partial patient anatomy known from a planning CT scan of the patient. Previous studies of secondary neutron doses have been performed with the GEANT4 Monte Carlo code ${ }^{6,7}$ or the Monte Carlo code MCNPX. ${ }^{8,9}$ It is important that correct implementation of these codes is validated with experimental studies on clinical proton beam lines.

The primary objective of this work is to measure the secondary neutron field produced by an uncollimated proton pencil beam impinging on different tissue-equivalent phantom materials using organic scintillation detectors. Additionally, the Monte Carlo code MCNPX-PoliMi was used to simulate
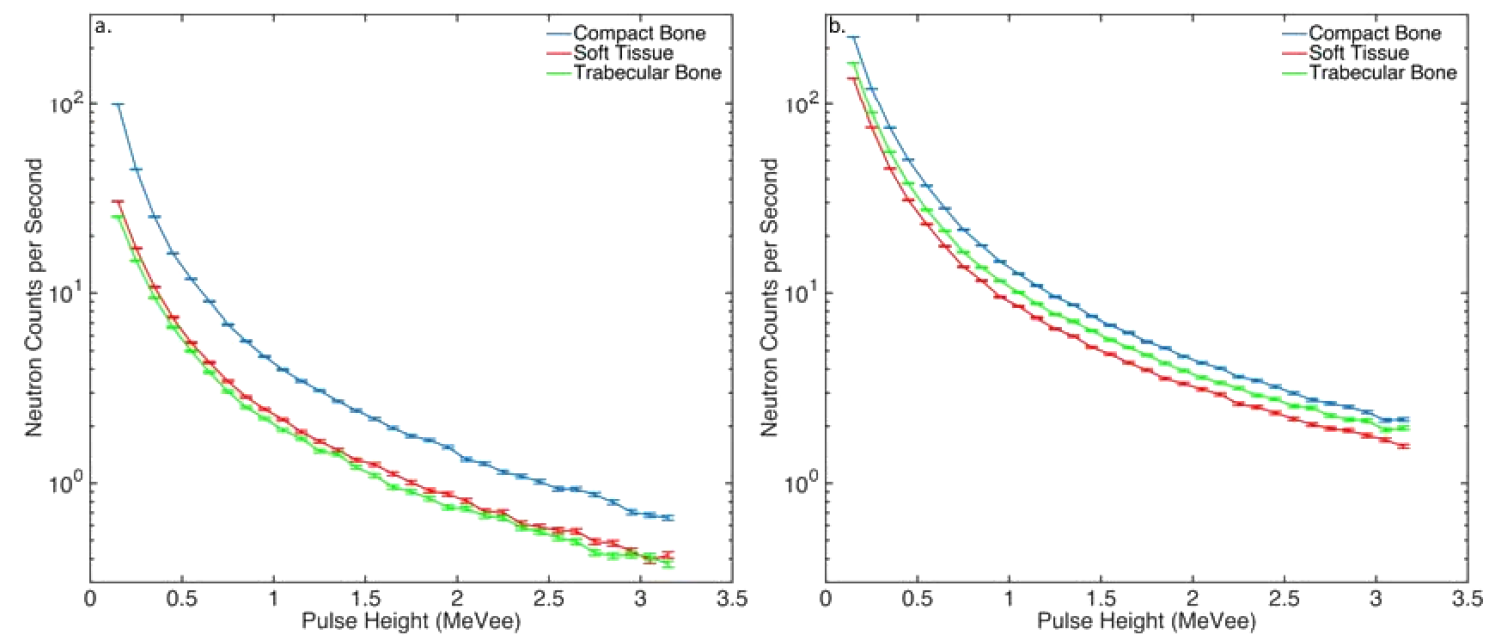

FIG. 3. Measured neutron pulse height distributions for three phantom target materials irradiated with (a) $155-\mathrm{MeV}$ and (b) $200-\mathrm{MeV}$ protons. 
TABLE I. The composition and density of the four CIRS, Inc., phantom materials used in the measurements taken at Loma Linda University Medical Center. Material composition is given in weight percent.

\begin{tabular}{lccc}
\hline \hline & Soft tissue $1.055 \mathrm{~g} / \mathrm{cm}^{3}$ & Trabecular bone $1.160 \mathrm{~g} / \mathrm{cm}^{3}$ & Compact bone $1.910 \mathrm{~g} / \mathrm{cm}^{3}$ \\
\hline Hydrogen & 8.47 & 6.99 & 3.30 \\
Carbon & 57.44 & 56.29 & 25.37 \\
Nitrogen & 1.65 & 2.03 & 0.91 \\
Oxygen & 24.59 & 22.72 & 35.28 \\
Magnesium & 7.62 & & 3.36 \\
Phosphorous & & 3.30 & 8.82 \\
Chlorine & 0.19 & 0.16 & 0.03 \\
Calcium & & 8.49 & 22.91 \\
\hline \hline
\end{tabular}

the detector response for comparison to the measured data. Comparison of the measured and simulated data was performed to validate this approach for monitoring secondary neutron dose during proton therapy.

\section{DESCRIPTION OF THE EXPERIMENT}

An experiment was performed at the Loma Linda University Medical Center proton therapy research beam line. Pulsed proton beams with a cycle time of about $2 \mathrm{~s}$ and an active spill length between 0.3 and $0.5 \mathrm{~s}$ were used to irradiate a variety of radiation therapy phantoms at energies of 155 and $200 \mathrm{MeV}$; the beam diameter was $2 \mathrm{~cm}$ at the exit of the vacuum tube and approximately $4 \mathrm{~cm}$ at the entrance of the phantom blocks. The beam current monitor in the research beam line was not operational and no ion chamber was available, so the total dose delivered to the blocks is unknown. The secondary particles were detected using two 3-in. diameter by 3-in. thick EJ-309 organic liquid scintillators. ${ }^{10}$ These detectors are each sensitive to fast neutrons and gamma rays. The signal from each detector is independently collected with a fast photomultiplier tube, with a time resolution of approximately $1.0 \mathrm{~ns}$. Pulse shape discrimination (PSD) was used to classify each detected pulse as either a neutron or a gamma ray, which allows selective analysis of the incident energy spectra. The PSD algorithm relies on integration of the individual pulses from the detectors; the integration range used for PSD determines the effective dead time of the cells, in this case approximately 300 ns.

Four tissue-equivalent phantom materials manufactured by Computerized Imaging Reference Systems (CIRS), Inc., Norfolk, VA were irradiated: compact bone, soft tissue, plastic water, and trabecular bone. Each of the phantoms was $30 \times 30 \mathrm{~cm}$ in the transverse dimension; the thickness varied from 18 to $30 \mathrm{~cm}$, but in each experiment was thick enough to completely stop the proton beam. The proton beams were aligned $5 \mathrm{~cm}$ from the detector-facing edge of the phantom being irradiated. Irradiations were performed for 20 min with $200-\mathrm{MeV}$ beam and $30 \mathrm{~min}$ with the $155-\mathrm{MeV}$ beam. Data were acquired using a digital measurement system developed by the University of Michigan Detection for Nuclear Nonproliferation Group based on a CAEN DT5720 waveform digitizer (12 bit, $250 \mathrm{MHz}$ ). Figure 1 shows a photograph of the experimental setup; the beam direction is left-to-right. The detectors were located $70 \mathrm{~cm}$ from the target perpendicular to the beam line.

The two detectors were gain-matched using a ${ }^{137} \mathrm{Cs}$ source, aligning the Compton edge to $300 \mathrm{mV}$. A detection threshold of $80 \mathrm{keV}$-electron-equivalent (keVee) was applied, which is defined as the amount of scintillation light that is emitted when a photon deposits $80 \mathrm{keV}$ on an electron in the scintillator. For neutrons, this threshold corresponds to

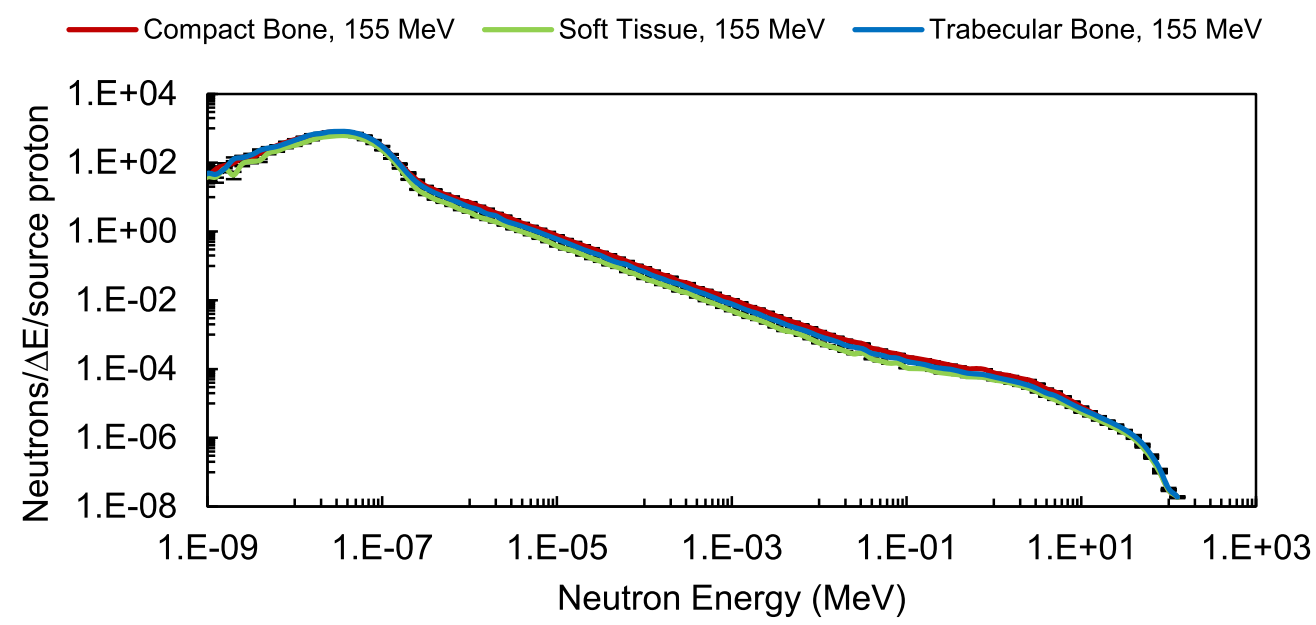

FIG. 4. The MCNPX-PoliMi simulated spectrum of neutrons entering the detectors during irradiation of the CIRS, Inc., phantom materials with $155 \mathrm{MeV}$ protons. 


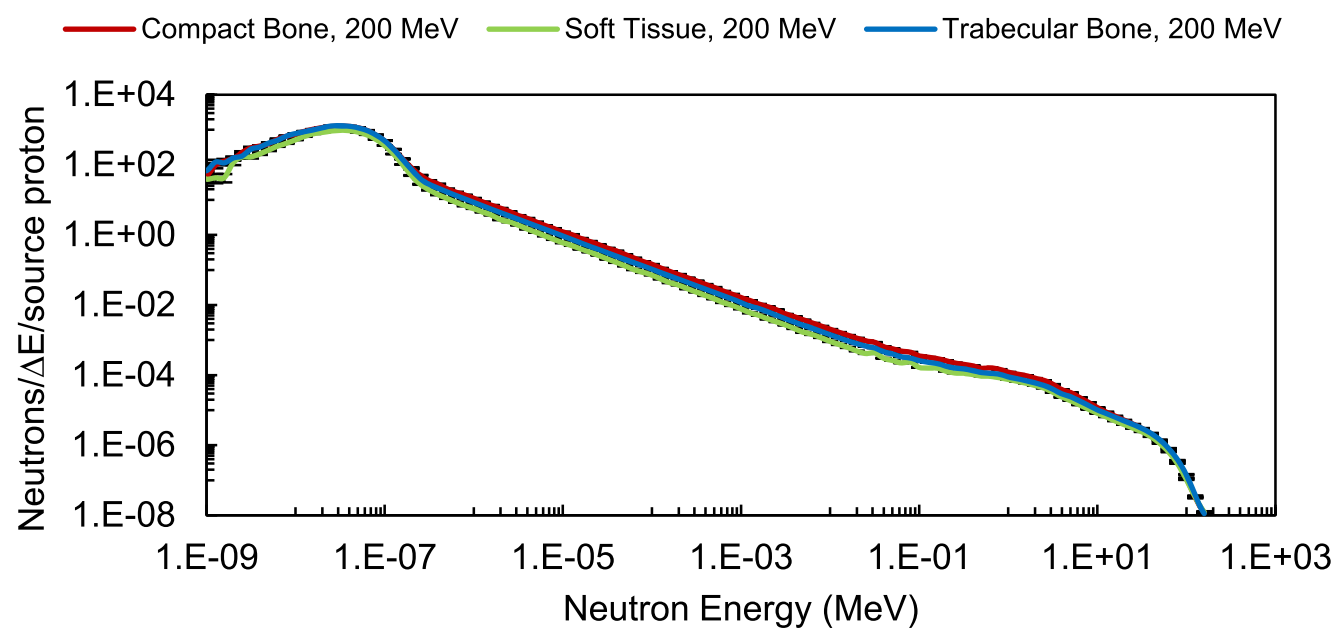

FIG. 5. The MCNPX-PoliMi simulated spectrum of neutrons entering the detectors during irradiation of the CIRS, Inc., phantom materials with $200 \mathrm{MeV}$ protons.

approximately $600 \mathrm{keV}$ of neutron energy deposited on a proton. ${ }^{11}$

The neutron pulses were distinguished from the gammaray pulses using an offline charge-integration method. ${ }^{12}$ The raw detector pulses were integrated over two regions: the tail region of the pulse and the total length of the pulse. The exact ranges of these "tail" and "total" integrals were optimized for each detector. Scintillation states excited by neutron scattering are longer lived than states excited by photon scattering. Consequently, detector pulses created by neutron events have larger tail integrals for a given total integral. Figure 2 shows the tail-integral versus total-integral projection of the soft tissue irradiation, which is indicative of the other results because the neutron and gamma-ray fields were all similar to one another. Despite the large number of photons present in the measured data, the neutron region is clearly separated from the photon region. A second-order discrimination line was fit between the two regions to discriminate the neutron and photon pulses: for a given total integral, any pulse whose tail integral falls above the discrimination line is called a neutron, and any pulse whose tail integral falls below the discrimination line is called a photon.

Figure 3 shows the resulting neutron pulse height distributions (PHDs) for the four phantom target materials irradiated with $155-\mathrm{MeV}$ and $200-\mathrm{MeV}$ protons.

\section{MONTE CARLO MODELING}

The MCNPX-PoliMi code was used to characterize the secondary neutrons produced during proton irradiation of biologically equivalent phantom materials. The code has the ability to write a collision-log file containing all information about the particle interactions inside of user-specified detector cells. These data are used to calculate detector response using a module-based postprocessing algorithm. ${ }^{13}$ The proton transport was performed using the ENDF proton data libraries included with the MCNPX code. ${ }^{14}$

The MCNPX-PoliMi model was used to simulate the proton irradiation experiment at the LLUMC experimental beam line. The floor and walls in the experimental hall were neglected in the model because they do not contribute significantly to measured quantities, and to increase computational efficiency. Table I lists the isotopic composition of the three CIRS, Inc., phantoms. Compact bone is the most-dense phantom material at $1.910 \mathrm{~g} / \mathrm{cm}^{3}$, followed by trabecular bone at $1.160 \mathrm{~g} / \mathrm{cm}^{3}$ and soft tissue at $1.055 \mathrm{~g} / \mathrm{cm}^{3}$.

The energy spectra of neutrons resulting from irradiation of the four CIRS, Inc., phantom materials were simulated using MCNPX-PoliMi. Figures 4 and 5 show the energy spectrum of neutrons entering the front faces of the two detectors upon irradiation of the CIRS, Inc., phantoms with 155 and $200 \mathrm{MeV}$

TABLE II. MCNPX-PoliMi simulated fluences of neutrons incident on the front faces of the two detectors for the different CIRS, Inc., phantom materials. Results are given as a ratio of neutrons incident on the front face of the detectors to the number of protons incident on the phantom.

\begin{tabular}{lcc}
\hline \hline Phantom material & $\begin{array}{c}\text { Incident proton } \\
\text { energy (MeV) }\end{array}$ & $\begin{array}{r}\text { Neutrons incident on detector } \\
\text { front face per incident proton }\end{array}$ \\
Soft tissue & 155 & $1.22 \times 10^{-4}$ \\
& 200 & $1.92 \times 10^{-4}$ \\
Trabecular bone & 155 & $1.23 \times 10^{-4}$ \\
& 200 & $1.95 \times 10^{-4}$ \\
Compact bone & 155 & $1.50 \times 10^{-4}$ \\
\end{tabular}




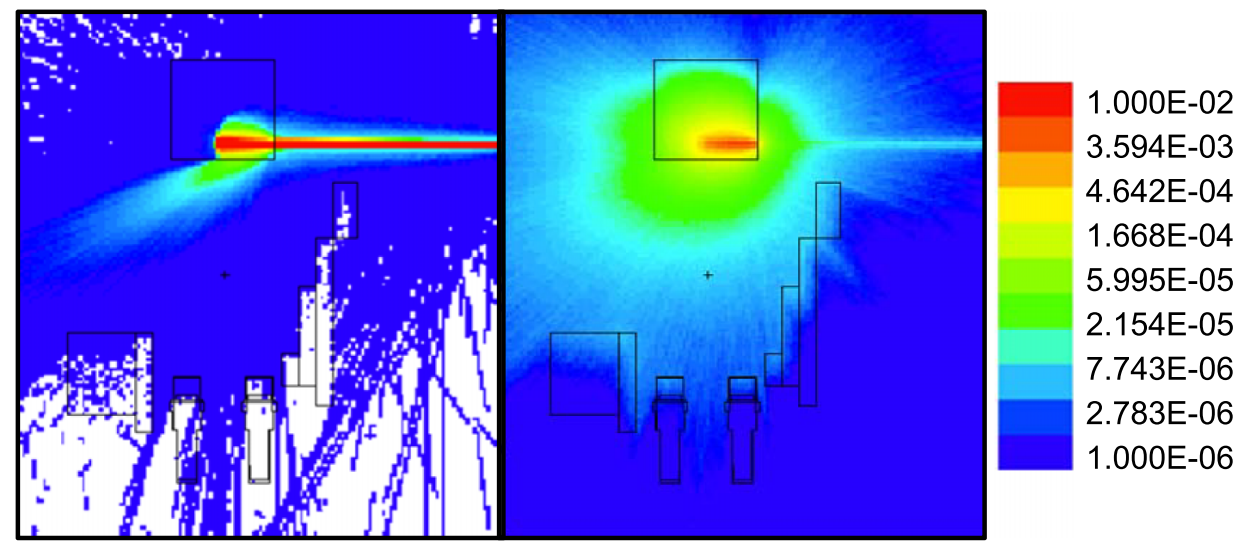

Fig. 6. MCNPX-PoliMi results show the proton (left) and neutron (right) flux distributions from $155-\mathrm{MeV}$ protons interacting in a soft tissue phantom; the units are protons (or neutrons) per $\mathrm{cm}^{2}$ per source proton.

protons, respectively. The shape of the neutron energy spectra is similar between the different phantom materials, which is consistent with the measured detector response shown in Fig. 3.

Table II lists the integral sum of the neutron energy spectra for all CIRS, Inc., phantom materials, the resulting value being the total number of neutrons entering the detectors upon irradiation of the phantom material with 155 or $200 \mathrm{MeV}$ protons. The neutron detector fluence was greater when phantom materials were irradiated with $200 \mathrm{MeV}$ protons compared to $155 \mathrm{MeV}$. The fluence of neutrons on the detectors was related to the density of the phantom material, with higher neutron fluence produced from denser phantom materials.

Figure 6 shows the proton- and neutron-flux distributions resulting from $155-$ and $200-\mathrm{MeV}$ protons, interacting with a soft tissue phantom; Fig. 7 shows the flux distributions for the $200-\mathrm{MeV}$ irradiation. These distributions were tallied in the 1-cm thick plane with respect to the center of the beam line. The proton distribution appears asymmetric because the beam was incident near the edge of the target phantom; protons that enter the air have a longer range than those transporting purely through the phantom. The proton flux distributions clearly show the Bragg peak at approximately $16 \mathrm{~cm}$ depth for $155 \mathrm{MeV}$ and $25 \mathrm{~cm}$ depth for $200 \mathrm{MeV}$. The secondary neutrons appear as an isotropic-like source emitted from the target along the path of the beam. While the highenergy neutrons are certainly forward-directed, the low-energy neutrons are more isotropic; additionally, the scattering in the phantom will also create an isotropic distribution.

Proton range and depth-dose characteristics within the phantom materials were also evaluated using MCNPX-PoliMi; the results are shown for the CIRS, Inc., soft tissue phantom in Fig. 8 . The dose deposited by protons was tallied in volumetric slices of the phantom using an MCNPX energy deposition tally. The resulting distribution matches the Bragg peak shape, as anticipated. The occurrence of the Bragg peak just beyond the sharp drop in proton fluence is expected as a majority of a proton dose is deposited at the end of its path.

The simulated ranges of protons in the four CIRS, Inc., phantom materials were compared to the continuous-slowing down approximation (CSDA) range calculated using the Bragg-Kleeman rule, given in the following:

$$
R_{T}=R_{\text {ref }} \sqrt{\frac{A_{T}}{A_{\text {ref }}}},
$$

where $R_{T}$ is the CSDA range of a proton at a given energy into a composite material $T$ in units of $\mathrm{g} / \mathrm{cm}^{2}, R_{\text {ref }}$ is the known CSDA range in $\mathrm{g} / \mathrm{cm}^{2}$ of a proton with the same energy

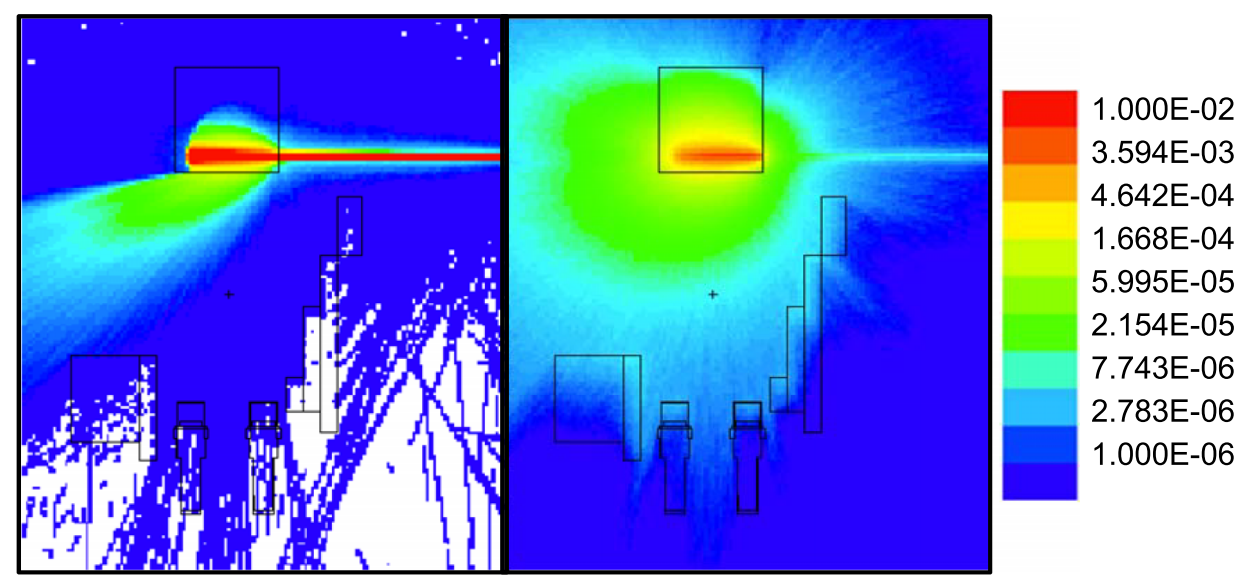

FIG. 7. MCNPX-PoliMi results show the proton (left) and neutron (right) flux distributions from 200-MeV protons interacting in a soft tissue phantom; the units are protons (or neutrons) per $\mathrm{cm}^{2}$ per source proton. 


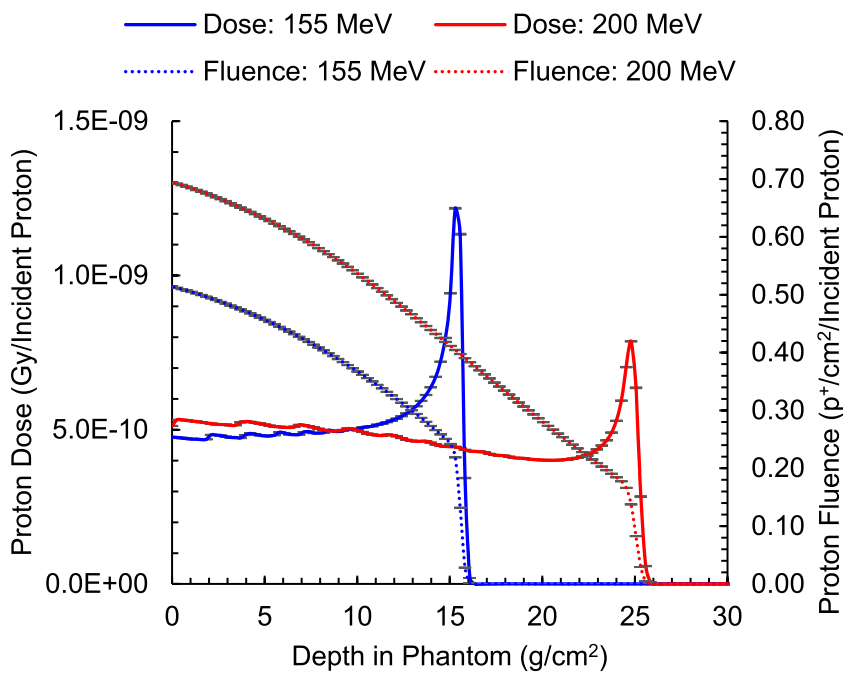

FIG. 8. The MCNPX-PoliMi simulated proton dose (solid lines) and fluence (dotted lines) as a function of depth in the CIRS, Inc., soft tissue phantom upon irradiation with $155 \mathrm{MeV}$ (blue) and $200 \mathrm{MeV}$ (red) protons.

into a reference material, and $A_{T}$ and $A_{\text {ref }}$ are the effective atomic numbers of the composite material $T$ and the reference material, respectively. These values are calculated using the following:

$$
\sqrt{A_{T}}=\left(\sum_{i} \frac{W_{i}}{\sqrt{A_{i}}}\right)^{-1},
$$

where $W_{i}$ is the mass fraction of the $i$ th element within the composite material and $A_{i}$ is the atomic number of that $i$ th element. ${ }^{15}$

Table III gives the required quantities for calculation of proton CSDA ranges in the four CIRS, Inc., materials. Reference materials were selected from those available in the National Institute of Standards and Technology (NIST) PSTAR database; selection was based on compositional similarity to the CIRS, Inc., phantom materials. The ICRU compact bone was chosen as the reference material for CIRS, Inc., compact bone, A-150 tissue-equivalent plastic as the reference material for CIRS, Inc., soft tissue and CIRS, Inc., trabecular bone, and finally polymethyl methacrylate (PMMA) for CIRS, Inc., plastic water. Along with compositional data, CSDA ranges of reference materials were gathered from the NIST PSTAR database. ${ }^{16}$

Table IV gives the CSDA ranges of the CIRS, Inc., phantom materials calculated using Eq. (2) along with the ranges simulated using MCNPX-PoliMi. Comparison gave good agree-
TABLE IV. The CSDA ranges of protons in the four CIRS, Inc., phantom materials with incident energy $E_{p}$ were calculated with the Bragg-Kleeman scaling rule given in Eq. (1). Reference materials were taken from those available in the NIST PSTAR database. The calculated CSDA range is compared to the proton range simulated by MCNPX-PoliMi.

\begin{tabular}{lcccc}
\hline \hline \multirow{2}{*}{ CIRS material } & $\begin{array}{c}\text { Incident proton } \\
\text { energy }(\mathrm{MeV})\end{array}$ & \multicolumn{3}{c}{ CSDA range $\left(\mathrm{g} / \mathrm{cm}^{2}\right)$} \\
\cline { 3 - 5 } Soft tissue & 155 & 17.50 & 17.68 & 1.0 \\
& 200 & 27.22 & 27.30 & 0.3 \\
Trabecular bone & 155 & 18.31 & 17.59 & 4.0 \\
& 200 & 28.48 & 27.65 & 2.9 \\
Compact bone & 155 & 19.80 & 19.42 & 1.9 \\
& 200 & 30.75 & 30.24 & 1.7 \\
\hline \hline
\end{tabular}

ment between the calculated and simulated ranges despite the differences in atomic composition between CIRS, Inc., materials and the reference materials. The largest discrepancy occurred between the calculated and simulated values in trabecular bone, likely due to the lack of materials in the NIST PSTAR database that had composition and density similar to those of the CIRS, Inc., trabecular bone phantom.

Figures 9 and 10 show the MCNPX-PoliMi simulated fluence map of neutrons within different energy groups upon irradiation of the CIRS, Inc., soft tissue phantom with 155 and $200 \mathrm{MeV}$ protons, respectively. Secondary neutrons between 0 and $5 \mathrm{MeV}$ are emitted isotropically, while those above $5 \mathrm{MeV}$ are more forward directed. Thus, the secondary neutron field can be separated into two distinct components; the isotropic, low-energy component and the forward-directed, high-energy component.

The biological effectiveness of neutron radiation peaks at energies of $1 \mathrm{MeV},{ }^{17}$ suggesting that the majority of the biologically weighted dose from secondary neutrons arises from the low-energy component of the secondary neutron field. It is evident from Figs. 9 and 10 that this dose will primarily be deposited within the patient.

\section{COMPARISON OF MEASURED AND SIMULATED DATA}

Tallies in the standard MCNP code calculate average energy deposition with linear response functions; however, the response of organic scintillators is nonlinear and depends on the exact details of the neutron collision history in the

TABLE III. Material densities, effective atomic numbers, and CSDA ranges of $155-$ and 200-MeV protons in NIST reference materials.

\begin{tabular}{lcccr}
\hline \hline Reference material & $\begin{array}{c}\text { Density } \\
\left(\mathrm{g} / \mathrm{cm}^{3}\right)\end{array}$ & $\begin{array}{c}\text { Effective } \\
\text { atomic } \\
\text { number }\end{array}$ & \multicolumn{2}{c}{ CSDA range $\left(\mathrm{g} / \mathrm{cm}^{2}\right)$} \\
\cline { 4 - 5 } A-150 tissue-equivalent plastic & 1.127 & 4.73 & 16.56 & $200 \mathrm{MeV}$ \\
Polymethyl methacrylate & 1.190 & 5.20 & 17.14 & 25.75 \\
ICRU compact bone & 1.850 & 6.76 & 17.94 & 26.64 \\
\hline \hline
\end{tabular}




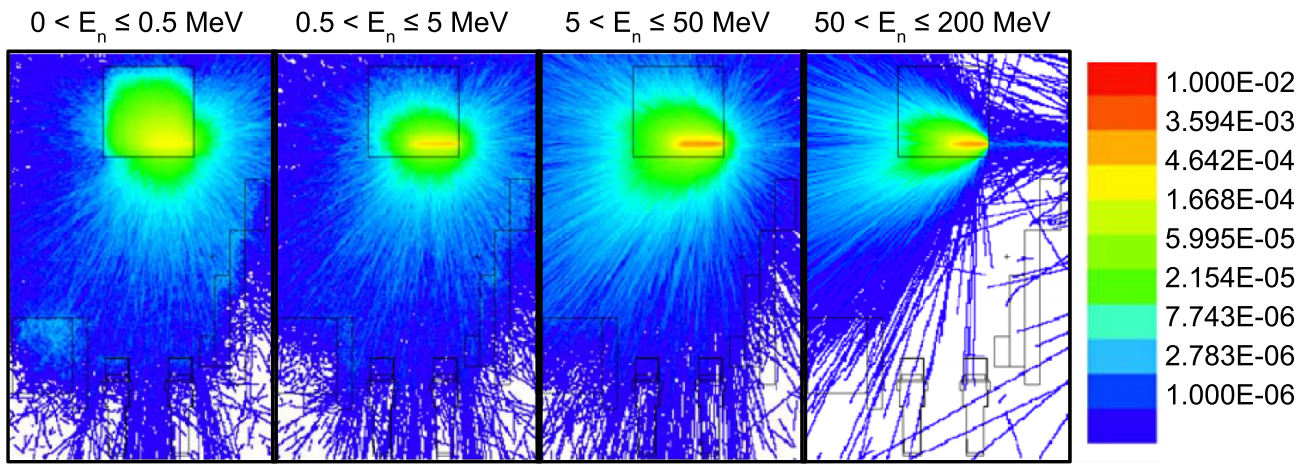

FIG. 9. MCNPX-PoliMi simulation of the fluence of neutrons with energy $E_{n}$ resulting from the CIRS soft tissue phantom irradiated with $155 \mathrm{MeV}$ protons, given in neutrons per $\mathrm{cm}^{2}$ per incident proton.

detector. ${ }^{18}$ The response of the EJ-309 scintillators was calculated using the MPPost code, ${ }^{19}$ which is a detection postprocessor distributed with the MCNPX-PoliMi code. Figure 11 shows the total simulated and measured neutron pulse height distribution from the EJ-309 scintillators. One should note that the measured distributions were arbitrarily scaled because the absolute proton fluence was not monitored during the experiment.

The pulse height distributions from the detectors can be converted into dose rate by inverting the known detector response functions. ${ }^{20}$ The detector response functions have been measured and previously published in Ref. 11. The energy deposited in the detector is converted to dose deposited (in gray) by dividing by the mass of each detector cell. This dose is then converted to dose equivalent with the ICRP21 energy-dependent radiation weighting factors. ${ }^{17}$ Table V summarizes the dose rates for each of the phantom materials and proton beam energies. For comparison, MCNPX (Ref. 14) point-detectors tallies with an ICRP-21 dose modifier are used to calculate neutron dose equivalent directly from simulated protons; for these calculations, all neutrons above the detection threshold were tallies. As expected, the dose rates unfolded from the detector response slightly under-predict the MCNPX calculations due to the limited sensitivity of our detection system to high-energy neutrons. These results show that the neutron dose rates at the detector position will reach significant levels at expected proton clinical intensities, which may be on the order of $10^{10}-10^{11} \mathrm{~s}^{-1}$.

\section{SUMMARY AND DISCUSSION}

Organic scintillators detect incident fast neutrons primarily through elastic scattering on hydrogen nuclei: neutrons with energy less than a few hundred $\mathrm{keV}$ cannot deposit sufficient energy to create a detectable pulse. For the experimental configuration considered in this work, approximately $37 \%$ of neutrons incident on the detectors have energy below $600 \mathrm{keV}$ and thus cannot be detected. This energy spectrum is related to the detector placement relative to the target block and selfattenuation of the neutrons within the target block, as well as neutron scattered through the sides and back of the detector assembly. We are currently investigating analysis techniques to enable data acquisition at lower detection thresholds, down to approximately $100 \mathrm{keV}$. A thermal detection medium such as ${ }^{6} \mathrm{Li}$ glass could be incorporated into the detection system to increase sensitivity to lower energy neutrons.

The neutrons produced by such high-energy protons will also be quite high in energy; in fact, they can take any energy up to the initial proton energy; approximately $6 \%$ of the neutrons incident on the detector are above $20 \mathrm{MeV}$. Because the elastic scattering cross section decreases as neutron energy increases, it is important to investigate the overall detection efficiency of the high-energy neutrons. Figure 12 shows the simulated energy spectrum of the neutrons incident on the detector face for $200-\mathrm{MeV}$ proton irradiation of the soft tissue target, as well as the portion of this incident spectrum that is detected. As expected, the detection efficiency decreases

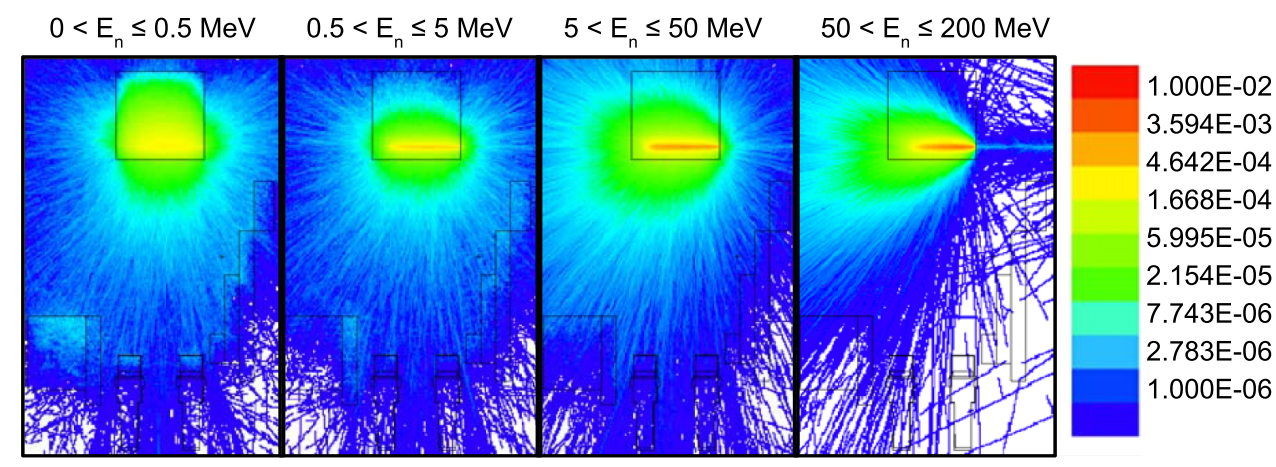

FIG. 10. MCNPX-PoliMi simulation of the fluence of neutrons with energy $E_{n}$ resulting from the CIRS, Inc., soft tissue phantom irradiated with $200 \mathrm{MeV}$ protons, given in neutrons per $\mathrm{cm}^{2}$ per incident proton. 


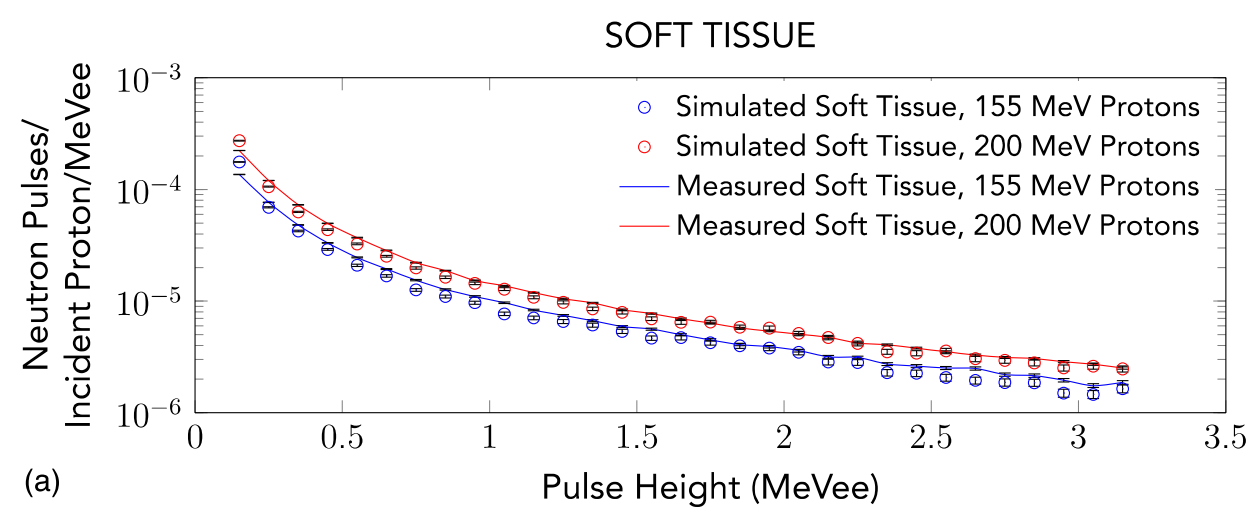

TRABECULAR BONE

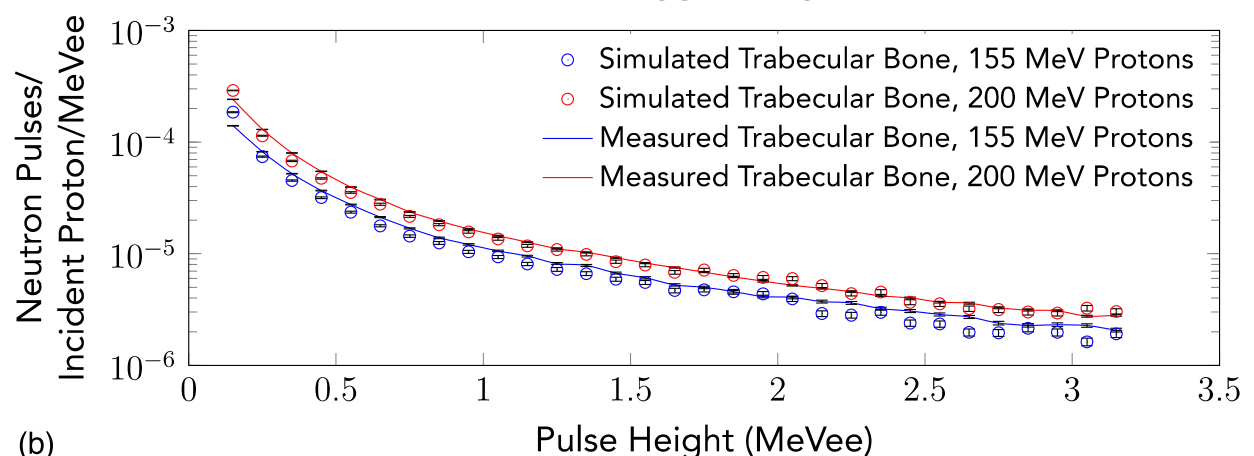

(b)

\section{COMPACT BONE}

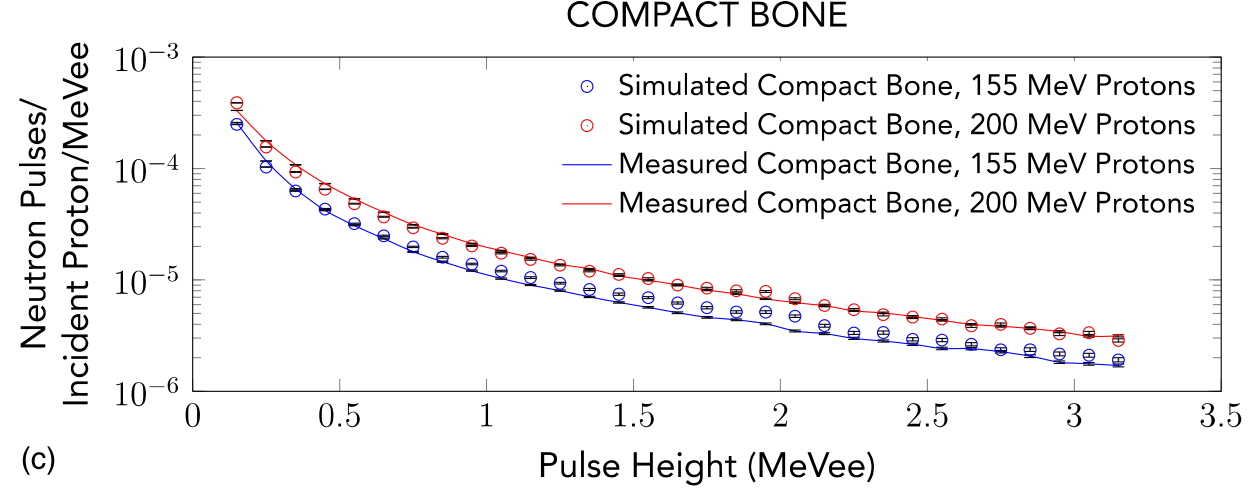

FIG. 11. MCNPX-PoliMi simulated and measured neutron pulse height distributions from 155- and 200-MeV irradiation of the CIRS, Inc., phantom materials (a) soft tissue; (b) trabecular bone; (c) and compact bone phantoms. Measured PHDs were arbitrarily scaled for comparison.

as the incident neutron energy increases; Fig. 12(b) shows the energy-dependent intrinsic neutron detection efficiency. The error bars on the plot are only statistical; uncertainties in the nuclear data particularly at high energies could contribute to the observed fluctuations. The overall intrinsic detection efficiency is approximately $15 \%$; however, if one considers only the portion of the incident spectrum that is above the detection threshold, the intrinsic efficiency is approximately

TABLE V. Dose rates at the detector positions calculated using a MCNP point detector tally as well as deconvolved from the simulated pulse height distributions. Units are mrem per hour per incident proton rate $\left(\mathrm{s}^{-1}\right)$.

\begin{tabular}{lccc}
\hline \hline & $\begin{array}{c}\text { Energy } \\
(\mathrm{MeV})\end{array}$ & $\begin{array}{c}\text { Unfolded detector } \\
\text { response }\end{array}$ & $\begin{array}{c}\text { McNPx point detector } \\
\text { tally }\end{array}$ \\
\hline Phantom material & 155 & $1.58 \times 10^{-7}$ & $2.71 \times 10^{-7}$ \\
Soft tissue & 200 & $2.41 \times 10^{-7}$ & $4.62 \times 10^{-7}$ \\
& 155 & $1.66 \times 10^{-7}$ & $2.66 \times 10^{-7}$ \\
Trabecular bone & 200 & $2.52 \times 10^{-7}$ & $4.58 \times 10^{-7}$ \\
& 155 & $2.17 \times 10^{-7}$ & $3.10 \times 10^{-7}$ \\
Compact bone & 200 & $3.27 \times 10^{-7}$ & $5.37 \times 10^{-7}$ \\
\hline \hline
\end{tabular}



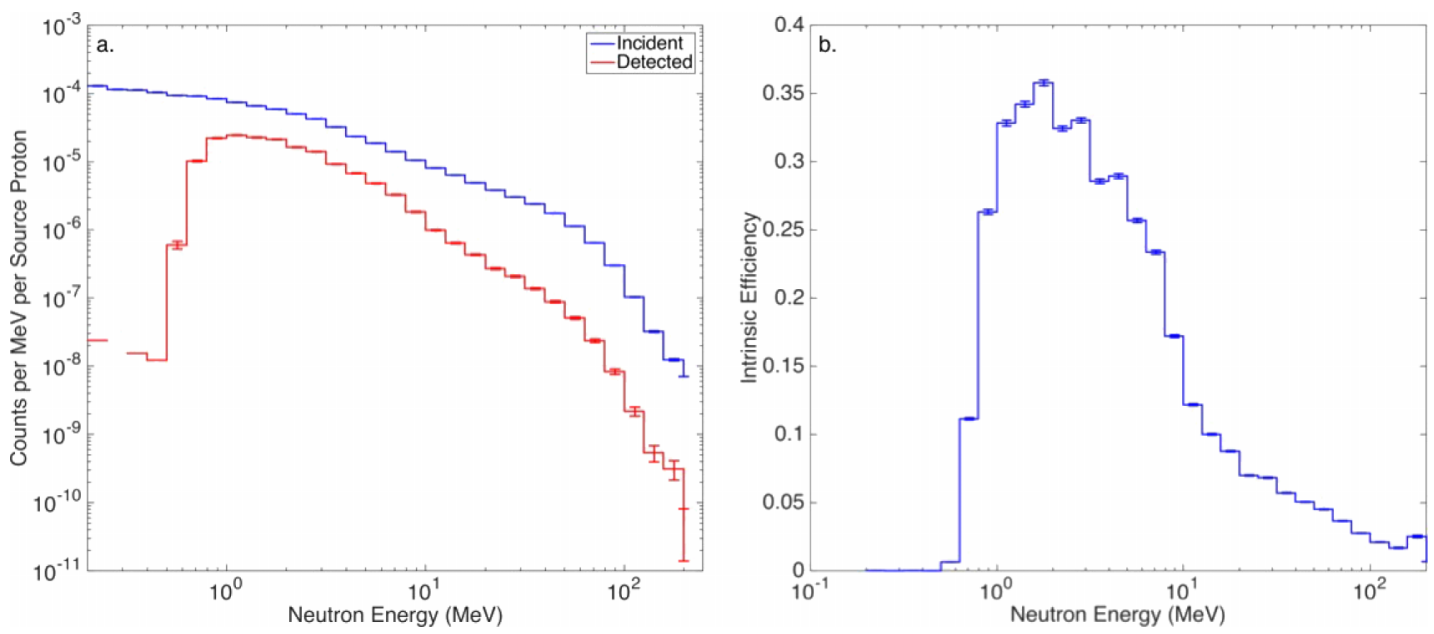

FIG. 12. (a) Simulated energy spectrum of incident and detected neutrons for 200-MeV proton irradiation of the soft tissue target; (b) energy dependent neutron detection efficiency.

$22 \%$. Furthermore, the radiation weighting factor peaks near $1 \mathrm{MeV}$ and begins to quickly decrease for higher energies.

\section{CONCLUSIONS}

We have demonstrated a method of monitoring the neutron component of the secondary radiation field produced by therapeutic protons. The method relies on direct detection of secondary neutrons and gamma rays using organic scintillation detectors. These detectors are sensitive over the full range of biologically relevant neutron energies above $0.5 \mathrm{MeV}$ and allow effective discrimination between neutron and photon dose.

An experiment was performed at the Loma Linda University Medical Center proton therapy research beam line and corresponding models were created using the MCNPX-PoliMi code. Our analysis showed agreement between the shape of the simulated and measured detector response. Once fully validated, simulated detector response can be used to assess neutron dose on a particular beam line without the need for experiments. Because the detector system is portable and sensitive to neutrons and gamma rays, the described system could be used in the future to evaluate secondary doses on various clinical beam lines for commissioning and prospective data collection in pediatric patients treated with proton therapy. Future work will focus on absolute validation of the simulation models as well as deconvolving the detector response to produce absolute dose rate measurements for neutrons, as well as photons in a single instrument.

\section{ACKNOWLEDGMENTS}

This work was funded in part by the ANDANTE grant from the Project 7 Framework of the European Union. The authors would like to thank Vladimir Varchena from CIRS, Inc., for providing the phantom material compositions and the target slabs used in the measurement. The authors would like to thank the proton accelerator operator staff at Loma Linda
University Medical Center for their support before during the experimental run.

\section{CONFLICT OF INTEREST DISCLOSURE}

The authors have no COI to report.

${ }^{a)}$ Author to whom correspondence should be addressed. Electronic mail: clarkesd@umich.edu

${ }^{1} \mathrm{~S}$. Garwicz et al., "Second malignant neoplasms after cancer in childhood and adolescence: A population-based case-control study in the 5 nordic countries," Int. J. Cancer 88, 672-678 (2000).

${ }^{2}$ W. Dorr and T. Herrmann, "Second primary tumors after radiotherapy for malignancies, treatment-related parameters," Strahlenther. Onkol. 178, 357-362 (2002).

${ }^{3}$ Proton Therapy Physics, Series in Medical Physics and Biomedical Engineering, edited by H. Paganetti (CRC/Taylor \& Francis, Boca Raton, FL, 2012).

${ }^{4}$ J. Farah, V. Mares, M. Romero-Expósito, S. Trinkl, C. Domingo, and V. Dufek, "Measurement of stray radiation within a scanning proton therapy facility: EURADOS WG9 intercomparison exercise of active dosimetry systems," Med. Phys. 42, 2572-2583 (2015).

${ }^{5}$ C. Lee, J. L. Williams, C. Lee, and W. E. Bolch, "The UF series of tomographic computational phantoms of pediatric patients," Med. Phys. 32, 3537-3548 (2005).

${ }^{6}$ C. Z. Jarlskog, C. Lee, W. E. Bolch, X. G. Xu, and H. Paganetti, "Assessment of organ-specific neutron equivalent doses in proton therapy using computational whole-body age-dependent voxel phantoms," Phys. Med. Biol. 53, 693-717 (2008).

${ }^{7}$ B. S. Athar and H. Paganetti, "Neutron equivalent doses and associated lifetime cancer incidence risks for head \& neck and spinal proton therapy," Phys. Med. Biol. 54, 4907-4926 (2009).

${ }^{8}$ W. D. Newhauser et al., "The risk of developing a second cancer after receiving craniospinal proton irradiation,” Phys. Med. Biol. 54, 2277-2291 (2009).

${ }^{9}$ P. J. Taddei et al., "Stray radiation dose and second cancer risk for a pediatric patient receiving craniospinal irradiation with proton beams," Phys. Med. Biol. 54, 2259-2275 (2009).

${ }^{10}$ Eljen Technologies, "EJ-309 liquid scintillator," http://www.eljentechnolo gy.com/index.php/products/liquid-scintillators/73-ej-309, accessed 18 June 2015.

${ }^{11}$ A. Enqvist, C. C. Lawrence, B. M. Wieger, S. A. Pozzi, and T. N. Massey, "Neutron light output response and resolution functions in EJ-309 liquid scintillation detectors," Nucl. Instrum. Methods Phys. Res., Sect. A 715, 79-86 (2013). 
${ }^{12}$ M. Flaska, M. Faisal, D. D. Wentzloff, and S. A. Pozzi, "Influence of sampling properties of fast-waveform digitizers on neutron-gamma-ray, pulseshape discrimination for organic scintillation detectors," Nucl. Instrum. Methods Phys. Res., Sect. A 729, 456-462 (2013).

${ }^{13}$ S. A. Pozzi, S. D. Clarke, W. Walsh, E. C. Miller, J. Dolan, M. Flaska, B. M. Wieger, A. Enqvist, E. Padovani, J. K. Mattingly, D. Chichester, and P. Peerani, "MCNPX-polimi for nuclear nonproliferation applications," Nucl. Instrum. Methods Phys. Res., Sect. A 694, 119-125 (2012).

${ }^{14}$ MCNPX Manual Version 2.7.0. LA-CP-11-00438 edited by D. B. Pelowitz, April 2011.

${ }^{15}$ G. F. Knoll, "Radiation interactions," in Radiation Detection and Measurement, 4th ed., edited by N. J. Hoboken (John Wiley, New York, NY, 2010), pp. 38-41.

${ }^{16}$ M. J. Berger, J. S. Coursey, M. A. Zucker, and J. Chang, "Estar, pstar, and ASTAR: Computer programs for calculating stopping-power and range tables for electrons, protons, and helium ions (version 1.2.3)," available http://physics.nist.gov/Star (National Institute of Standards and Technology, Gaithersburg, MD, 2005).

${ }^{17}$ ICRP Committee 3 Task Group, P. Grande, and M. C. O'Riordan, "Data for protection against ionizing radiation from external sources: Supplement to ICRP publication 15," in ICRP-21, International Commission on Radiological Protection (Pergamon, Oxford, UK, 1971).

${ }^{18}$ S. A. Pozzi, M. Flaska, A. Enqvist, and I. Paszit, "Monte Carlo and analytical models of neutron detection with organic scintillation detectors," Nucl. Instrum. Methods Phys. Res., Sect. A 582, 629-637 (2007).

${ }^{19}$ E. C. Miller, S. D. Clarke, M. Flaska, S. A. Pozzi, and E. Padovani, "MCNPXpolimipost-processing algorithm for detector response simulations,' J. Nucl. Mater. Manage. XL(2), 34-41 (2011).

${ }^{20}$ C. A. Miller, S. D. Clarke, and S. A. Pozzi, "WE-AB-BRB-11: Portable fast neutron and photon dose meter," Med. Phys. 42, 3652 (2015). 